\title{
Modeling and simulation of high-speed milling centers dynamics
}

\author{
El Bechir Msaddek • Zoubeir Bouaziz • Maher Baili • \\ Gilles Dessein
}

Received: 9 June 2010 / Accepted: 3 August 2010 / Published online: 29 August 2010

(C) The Author(s) 2010. This article is published with open access at Springerlink.com

\begin{abstract}
High-speed machining is a milling operation in industrial production of aeronautic parts, molds, and dies. The parts production is being reduced because of the slowing down of the machining resulting from the tool path discontinuity machining strategy. In this article, we propose a simulation tool of the machine dynamic behavior, in complex parts machining. For doing this, analytic models have been developed expressing the cutting tool feed rate. Afterwards, a simulation method, based on numerical calculation tools, has been structured. In order to validate our approach, we have compared the simulation results with the experimental ones for the same examples.
\end{abstract}

Keywords Machining $\cdot$ Pocket $\cdot$ Modeling $\cdot$ Simulation . HSM

E. B. Msaddek $(\square) \cdot Z$. Bouaziz

Unit of Research of Mechanics of the Solids,

Structures and Technological Development, ESSTT,

Tunis, Tunisia

e-mail: elbechir.msaddek@gmail.com

Z. Bouaziz

e-mail: zoubeir.bouaziz@enis.rnu.tn

M. Baili · G. Dessein

Laboratory Production Engineering,

National School of Engineers of Tarbes,

47 Avenue Azereix, BP 1629, 65016 Tarbes Cedex, France

M. Baili

e-mail: Maher.Baili@enit.fr

G. Dessein

e-mail: gilles.dessein@enit.fr

$\begin{array}{ll}\text { Nomenclature } \\ V_{\mathrm{f}}(t) & \text { Instantaneous feed rate } \\ \vec{V} & \text { Feed rate vector } \\ V_{\mathrm{ft}} & \text { Tangential feed rate } \\ V_{\mathrm{fprog}} & \text { Programmed feed rate } \\ V_{\mathrm{ftcy}} & \text { Feed rate imposed by } t_{\mathrm{cy}} \\ V_{\mathrm{flsacc}} & \text { Feed rate for static look ahead imposed by the } \\ & \text { acceleration } \\ V_{\mathrm{flsjerk}} & \text { Feed rate for static look ahead imposed by the } \\ & \text { jerk } \\ V_{\mathrm{s}} & \text { Feed rate for static look ahead } \\ V_{\mathrm{st}} & \text { Feed rate for modified static look ahead } \\ V_{\mathrm{f}}(i) & \text { Feed rate of a block }(i) \\ V_{\max } & \text { Maximal feed rate } \\ V_{\max } i & \text { Maximal feed rate of axis }(i) \\ A(t), A_{\mathrm{f}} & \text { Instantaneous feed acceleration } \\ \vec{A} & \text { Feed acceleration vector } \\ A_{t} & \text { Tangential acceleration } \\ A_{n} & \text { Normal acceleration } \\ A_{\max } & \text { Maximal acceleration } \\ A_{\max } i & \text { Maximal acceleration of axis }(i) \\ J(t) & \text { Instantaneous feed Jerk } \\ \vec{J} & \text { Jerk vector } \\ J_{\mathrm{t}} & \text { Tangential Jerk } \\ J_{\mathrm{c}} & \text { Normal Jerk } \\ J_{\max } & \text { Maximal Jerk } \\ J_{\max } i & \text { Maximal Jerk of axis }(i) \\ J_{\text {curv }} & \text { Curvilinear tangential Jerk } \\ J_{\text {tcurv }} & \text { Tangential Jerk on curvature } \\ r_{\mathrm{jct}} & \text { Rate of curvilinear jerk associated of tangential } \\ t_{\mathrm{cy}} & \text { jerk } \\ \mathrm{TIT}(*) & \text { Time of interpolation cycle } \\ \delta t & \text { Interpolation tolerance of trajectory } \\ & \text { Crossing time of discontinuity } \\ & \end{array}$




$\begin{array}{ll}R, R(s) & \text { Curvature radius } \\ R_{j} & \text { Curvature radius of block }(j) \\ L, L_{\text {traj }} & \text { Length of the tool path } \\ L_{i} & \text { Length of the tool path of a block }(i) \\ \beta & \text { Angle between two blocks } \\ \beta_{j} & \text { Angle of a block }(j) \\ \alpha & \text { Angle between a block and the machine axis } \\ \alpha_{j} & \text { Angle between a block }(j) \text { and the machine axis } \\ d & \text { Distance } \\ d_{\mathrm{acc}} & \text { Acceleration distance } \\ d_{\mathrm{dec}} & \text { Deceleration distance }\end{array}$

\section{Introduction}

High-speed milling (HSM) has very interesting characteristics in the scope of the realization of high-quality mechanical parts in automobile industry and aeronautics [1]. The complex parts machining in HSM allows to take off the maximum material in the minimum time [2]. The cost of the part is then reduced [3], since the machining time represents an important part of the cost price.

The conception and the ordering of numerical drive tool machines necessitate the development of certain mathematical models [4]. The transformation models, between the work space and the dynamic models, defining the motion equations of the tool machine, allow the establishment of the relations between the couples or forces exerted by the actuators, and the positions, the speeds, and the acceleration of the axis articulations [5].

The geometrical shape influences the trajectories. The latter, characterized by speeds and accelerations, will be treated by the Numerical Controlled Unit (NCU) and will engage a certain imprecision (machine behavior) [6]. Thus, the dynamic modeling becomes a necessity for the machining optimization [7].

Recent studies have been interested in the HSM machines behavior modeling for pockets and complex shapes machining. Monreal et al. [4] and Tapie et al. [7] have treated the influence of the tool trajectory upon the machining time in HSM. Guardiola et al. [8] and Souza et al. [9] have evaluated the speed rate oscillation while machining and its influence upon the part obtaining time as well as upon its surface quality. Moreover, Souza et al. [9] have considered the influence of the tolerance value provided by the CAM software for the calculation of the desired tool trajectory.

Dugas [10] and Pateloup et al. [11, 12] have integrated the dynamic modeling of HSM machines by justifying the variation applied to the feed rate. Some parameters like the jerk, the acceleration, and the time of interpolation cycle have been used in order to make the machine slowing down in linear and circular interpolation explicit. Thus, Mawussi et al. [7] and Pateloup [13] have included the HSM machine real behavior study in the pocket hollowing out. Thus, other interpolations like B-spline are tested with the 840 D Siemens controller.

In most studies, the feed rate variation is not justified; this is one of the aims of this paper. Besides, there are no other studies which numerically examine and analyze the tool trajectory influence in CAM upon the HSM machine feed rate for the pockets hollowing out.

In this article, we present a feed rate calculation dynamic model according to the tool trajectory. This modeling includes the jerk, the acceleration, and the interpolation cycle time. Then, we simulate the feed rate for a pocket hollowing out and for a complex part machining with the 840 D Siemens controller.

In the first part, the detailed dynamic models development of both the axis and controller permits to better express the real behavior of the HSM tool machine. In the second part, the exploitation of the modeling for the real machining feed rate simulation is realized in the case of two parts of different shapes.

\section{The tool-machine NC dynamic modeling in HSM}

\subsection{Modeling step}

The dynamic modeling of the numerical drive HSM tool machine necessitates essentially the modeling of both the NCU controller and the motions axis.

The NCU behavior modeling is carried out through three steps. First of all, the integration of the connecting arcs is modeled. This is the phase of the tool trajectory preparation. Afterwards, the "Static Look Ahead" is also modeled. With this criterion, The NCU imposes the adequate speed in costume time, taking into account the axis capacities (axis modeling). Then, the "Dynamic Look Ahead" is modeled. It is the NCU capacity to anticipate in speed and acceleration during the machining to avoid the sudden acceleration (path overrun).

The modeling step is represented in the following diagram:

(Figure 1)

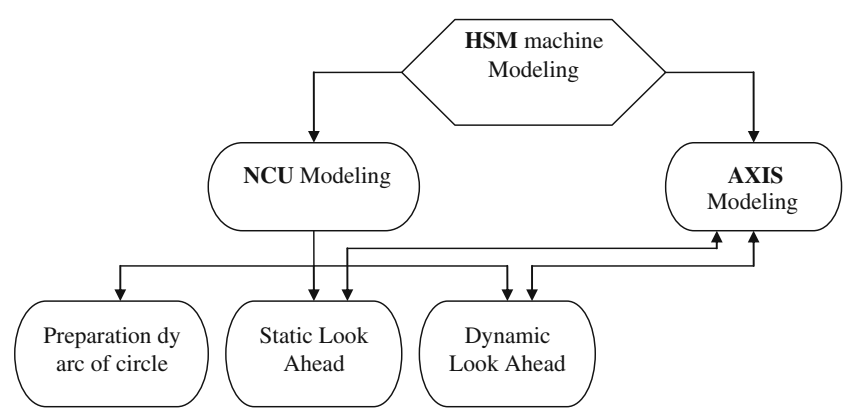

Fig. 1 A HSM machine dynamic modeling step 
Fig. 2 Tangential discontinuity and connecting with arcs of circle

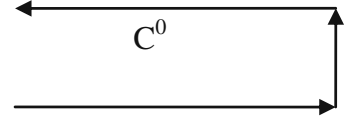

(a)

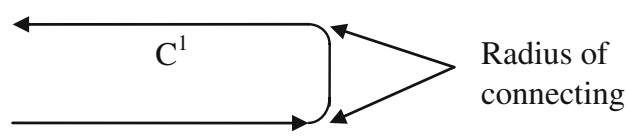

(b)

\subsection{NCU behavior modeling}

\subsubsection{Tool path modeling by arc of circle}

In $\mathrm{CAM}$, the tool path $\mathrm{C}^{0}$ (Fig. 2a) presents sharp angles while changing direction. In HSM machining, this sudden change necessitates the violent deceleration and the stopping of the machine for the crossing of tangential discontinuity. The NCU integrates connecting arcs in order to solve this problem. We obtain, then, the modified trajectory $\mathrm{C}^{1}$ with continuity in tangency (Fig. 2b).

Figure 3 presents the construction of connection radius between the three blocks $(i-1),(i)$, and $(i+1)$ of respective lengths $L_{i+1}, L_{i}$, and $L_{i-1}$. The radius expression $R_{j}$ of the connection between two blocks takes into account the angle of passage between the block $(i-1)$ and the block $(i) \beta_{j}$, of the trajectory interpolation tolerance TIT* and of the mini. Length between $L_{i}$ and $L_{i-1}[1]$.

$R_{j}=\operatorname{MIN}\left(\frac{\operatorname{TIT}^{*}}{\tan \left(\frac{\beta_{j}}{2}\right)} ; \frac{L}{2 \tan \left(\frac{\beta_{j}}{2}\right)}-\operatorname{TIT}^{*}\right)$ With $L=\operatorname{MIN}\left(L_{i} ; L_{i-1}\right)$

The passage of the tangential discontinuity maximal feed rate depends on the integrated arc radius. The arc radius $R$ leads to a more important speed limitation for the weak values of the $\beta$ angle and also to a less strong speed limit for the greater values of the same angle.

Considering that the arc of circle of a radius $R$ is crossed with a maximal tangential jerk $J_{\max }$ (derived from the acceleration), the maximal feed rate passage of the block transition is proportional to $J_{\max }$ [7].

This speed depends on the arc radius and so it depends on the Eq. 1 of the angle between the two segments $\beta$ and the tolerance TIT*.

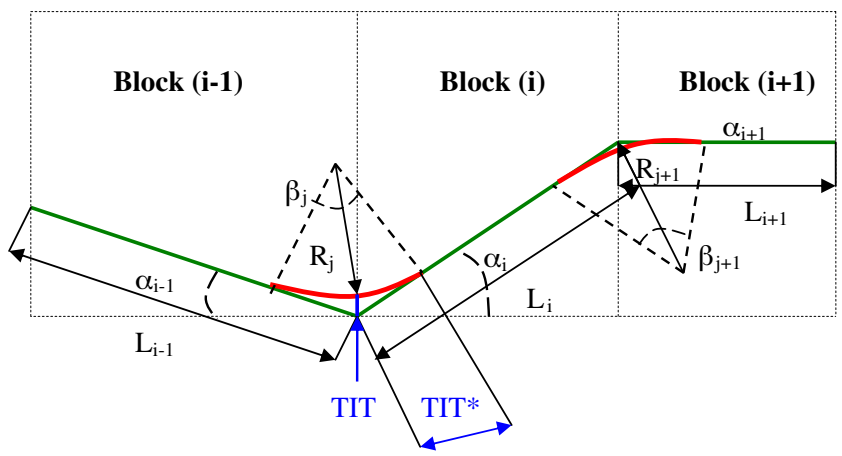

Fig. 3 Trajectory modeling by arc of circle
Consequently, the maxi jerk $J_{\max }$ depends on the tool path. In this case, we can have two types of configuration:

- Case of curvature discontinuity between a segment and an arc, $\mathrm{C}^{1}$ discontinuity (Fig. 4):

In this case, the jerk is according to the axis directions ( $\alpha$ : angle between the axis and the reference machine $\left(\overrightarrow{X_{m}}, \overrightarrow{Y_{m}}\right)$. The covered trajectory is ABCD which represents three blocks and a relative reference $(\vec{t}, \vec{n})$.

The maximal jerk at the point $\mathrm{B}$ is given by [7]:

$J_{\max }(B)=\min \left(\frac{J_{\max } X}{|\cos (\alpha)|} ; \frac{J_{\max } Y}{|\sin (\alpha)|}\right)$

- Case of curvature discontinuity between two arcs of circle of radius $R_{1}$ and $R_{2}$ connected in tangency, $\mathrm{C}^{2}$ discontinuity, (Fig. 5):

The crossing speed determination method of this discontinuity $V_{f}$, is as follows [12]:

The position $R(s)=R r_{1}$ à $t-\delta t$ et $R(s)=R r_{2}$ à $t+\delta t$

With $\delta t$ : time of the discontinuity crossing.

The tangential acceleration is nil on the curvature discontinuity. Well, the normal acceleration varies and can be written on the basis of Frenet $\overrightarrow{(n,} \vec{t})$ as follows:

$$
\begin{gathered}
\vec{A}\left(t+\frac{\delta t}{2}\right)-\vec{A}\left(t-\frac{\delta t}{2}\right)=\left(\frac{\left(R r_{1}-R r_{2}\right) \cdot V_{f}^{2}}{R r_{1} R r_{2}}\right) \vec{N}_{f} \\
=\left(j_{c \max } \cdot \delta t\right) \vec{N}_{f} \Rightarrow V_{f}=\sqrt{\frac{j_{c \max } \cdot \delta t \cdot R r_{1} \cdot R r_{2}}{\left|R r_{1}-R r_{2}\right|}}
\end{gathered}
$$

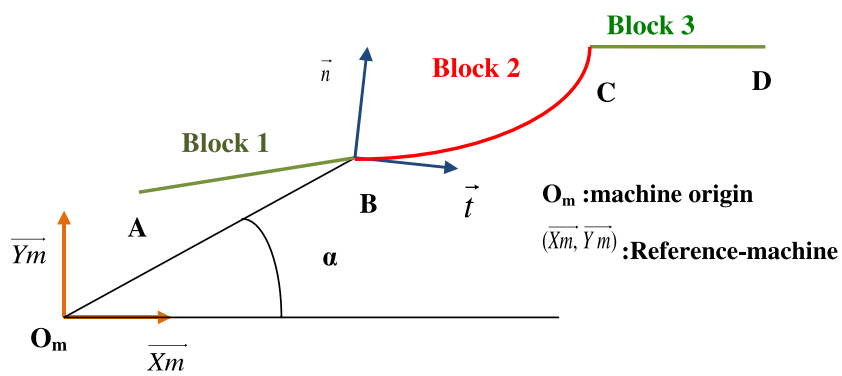

Fig. 4 Curvature discontinuity between two segments and an arc in the plan $(X, Y)[7]$ 
Fig. 5 Curvature discontinuity between two arcs of circle [12]

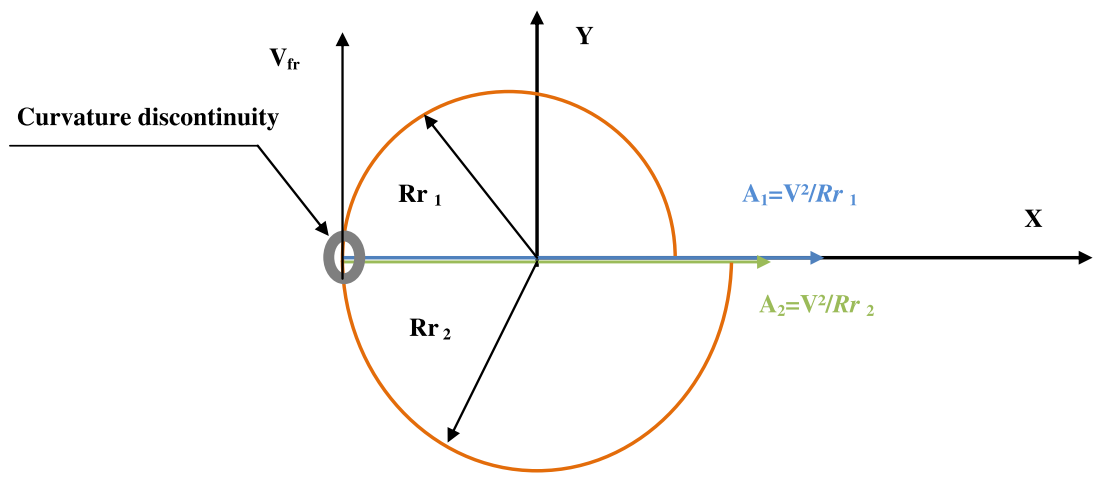

\subsubsection{Static look ahead}

The HSM machine has at its disposal the interpolation axis $\mathrm{X}, \mathrm{Y}$, and $\mathrm{Z}$ which are different at the level of their dynamic capacities. Each axis $[i]$ has a maximal speed $V_{\max } i$, a maximal acceleration $A_{\max } i$, and a maximum jerk $J_{\max } i$. These capacities depend on the engines and on the loads characteristics. In shape machining, we are then limited by the less dynamic (less rapid) axis $i$ :

$V_{\max }=\min \left(V_{\max } i\right), A_{\max }=\min \left(A_{\max } i\right)$, and $J_{\max }=\min \left(J_{\max } i\right)$

There are different types of feed rate limitations. If the trajectory is of class $\mathrm{C}^{0}$, the controller algorithm carries out the following calculations for each block:

$V_{\text {ftcy }}=\min \left(V_{\text {fprog }}, L / t_{\text {cy }}\right)$

This calculation puts into evidence the programmed speed $V_{\text {fprog }}$, the block length $L$, and the interpolation cycle time $t_{\mathrm{cy}}$. The block machining time must not be inferior to the $t_{\mathrm{cy}}$. So, the speed will be minimized if $L$ is very small $\left(t<t_{\mathrm{cy}}\right)$.

If the trajectory is of class $\mathrm{C}^{1}$, the limitations of speed in circular interpolation, in the plan $X Y$, are calculated for each angular position on the arc of circle of a radius $R$. In this way, the following calculations determine the most restrictive axis in speed, in acceleration, and in jerk:

$$
\begin{aligned}
& V_{\mathrm{ft}}=\min \left(\frac{V_{\max } X}{\left|\cos \left(\alpha_{i}\right)\right|} ; \frac{V_{\max } Y}{\left|\sin \left(\alpha_{i}\right)\right|}\right) \\
& \left.\left.A_{\mathrm{n}}=\min \left(\frac{A_{\max } X}{\left|\cos \left(\alpha_{i}\right)\right|} ; \frac{A_{\max } Y}{\left|\sin \left(\alpha_{i}\right)\right|}\right) \text { pour } \alpha_{i} \in\right] 0 . \beta\right] \\
& J_{\mathrm{t}}=\min \left(\frac{J_{\max } X}{\left|\cos \left(\alpha_{i}\right)\right|} ;\left(\frac{J_{\max } Y}{\left|\sin \left(\alpha_{i}\right)\right|}\right)\right) \\
& V_{\text {ftcy }}=\frac{R \times \beta}{t_{\mathrm{cy}}}
\end{aligned}
$$

The acceleration is composed of tangential acceleration $a_{\mathrm{t}}$ and of a normal one $a_{\mathrm{n}}$ in the basis of Frenet $\left.\overrightarrow{(n,} \vec{t}\right)$ [11].

$\vec{A}=\frac{\overrightarrow{d V_{\mathrm{f}}}}{d t}=\frac{d V_{\mathrm{f}}(t)}{d t} \cdot \vec{T}+\frac{V_{\mathrm{f}}^{2}(t)}{R} \cdot \vec{N}$

$A_{\mathrm{t}}=\frac{d V_{\mathrm{f}}(t)}{d t}$ and $A_{\mathrm{n}}=\frac{V_{\mathrm{f}}^{2}}{R}$

For a linear block $A_{\mathrm{t}}=\frac{d V_{\mathrm{f}}(t)}{d t}$ and $A_{\mathrm{n}}=0$

If the feed rate is constant, the corresponding acceleration is only normal $A_{\mathrm{n}}$ [14]. Then, so as the machine can follow a curve of a curvature radius $R$, at an imposed feed rate $V_{\mathrm{f}}$, we must necessarily have:

$A_{\mathrm{n}} \geq V_{\mathrm{f}}^{2} / R$

When this relation cannot be satisfied, the controller remakes the feed rate calculation in static look ahead linked to the acceleration $V_{\text {flsacc }}$, we have, then:

$V_{\text {flsacc }}=\sqrt{R \times A_{\mathrm{n}}}$

On the other hand, a small curvature radius induces a curvature jump and consequently an important couple of deceleration/acceleration accompanied by an infinite variation of jerk. In these different cases, a jerk limitation is used [11].

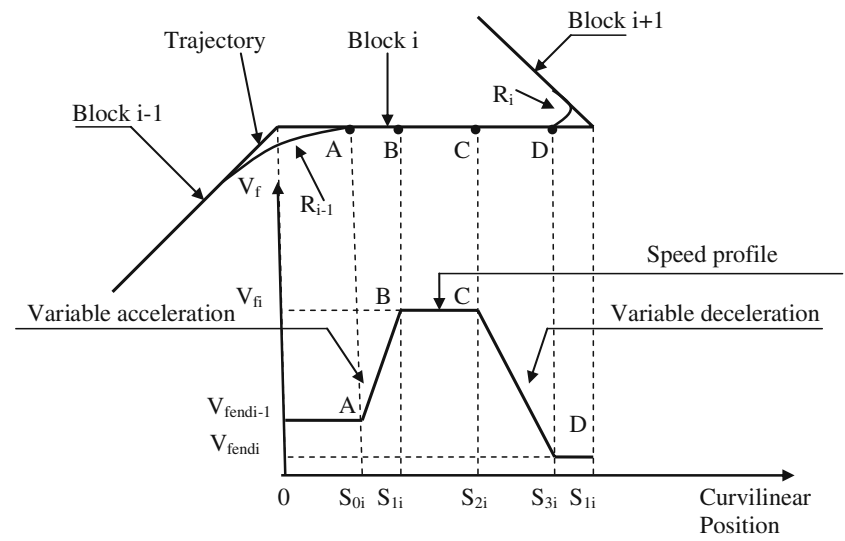

Fig. 6 Correspondence between the trajectory and the speed profile 


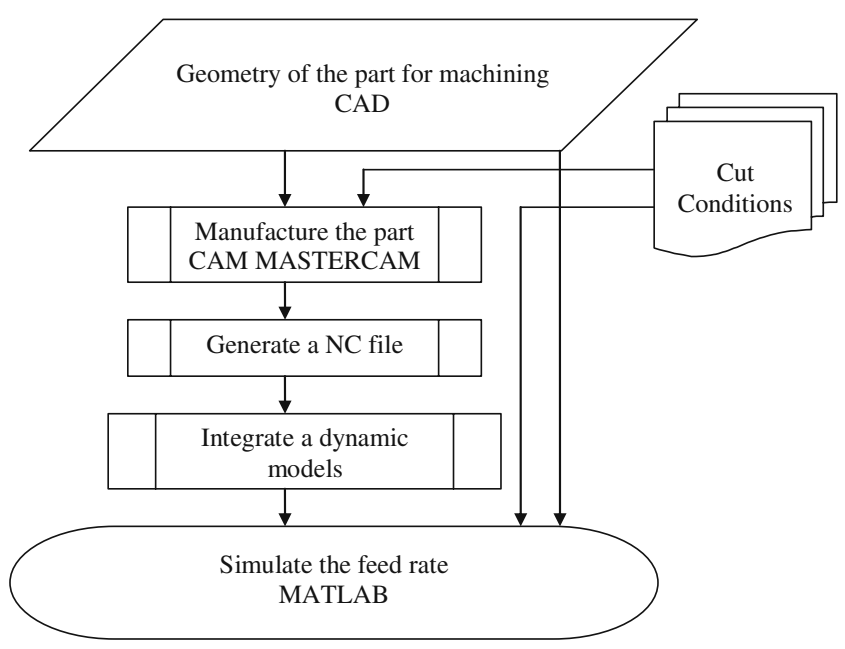

Fig. 7 Modelizing and simulation global step

The jerk is the acceleration derivative. Each acceleration component is controlled by a tangential jerk $J_{\mathrm{t}}$ and a centripetal jerk $J_{\mathrm{c}}$. In the stationary state of the speed $\frac{d A}{d t}=0$, Eq. 12 shows that the jerk is tangential $J_{\mathrm{t}}$. Hence, the maximal jerk $J_{\max i}$ of the axis $i$ limits the value of the tangential jerk $J_{\mathrm{t}}$.

$\vec{J}=\frac{d \vec{A}}{d t} \rightarrow \vec{J}=\frac{d A}{d t} \vec{N}+A \frac{d \vec{N}}{d t}=\vec{J}_{\mathrm{t}}$

According to (8) and (9):

$\vec{J}=\frac{d \vec{A}}{d t}=\frac{d A}{d t} \vec{N}+A \frac{d \vec{N}}{d t}=\frac{V_{\mathrm{f}}^{2}}{R} \cdot \frac{V_{\mathrm{f}}}{R} \cdot \vec{T}=\frac{V_{\mathrm{f}}^{3}}{R^{2}} \cdot \vec{T}=\overrightarrow{J_{\mathrm{t}}}$

For a feed rate $V_{\mathrm{f}}$ imposed, we must necessarily have:

$J_{\max }=J_{\mathrm{t}} \geq V_{\mathrm{f}}^{3} / R^{2}$

When this relation cannot be satisfied, the controller recalculates the feed rate in static look ahead linked to the jerk $V_{\text {flsjerk }}$, then we have:

$V_{\text {flstjerk }}=\sqrt[3]{J_{\mathrm{t}} \times R^{2}}$

Fig. 8 Tested trajectory [15]
The feed rate $V_{\mathrm{s}}$ representing the minimum different limitation types is called static look ahead.

$V_{\mathrm{s}}=\min \left(V_{\mathrm{fprog}}, V_{\mathrm{ftcy}}, V_{\mathrm{ft}}, V_{\mathrm{flsacc}}, V_{\mathrm{flstjerk}}\right)$

Tapis et al. [14] add the effects of the tangential jerk in curvature $J_{\text {tcurv }}$ and prove its limitation effect by the experimental. The tangential jerk in curvature is the product of the curvilinear tangential jerk $J_{\text {curv }}$ and of the rate of the curvilinear jerk associated to the tangential jerk $r_{\text {jct }}$.

$J_{\text {tcurv }}=J_{\text {curv }} \times r_{\text {jct }} \Rightarrow V_{\text {jtcurv }}=\sqrt[3]{J_{\text {tcurv }} \times R^{2}}$

Afterwards, the feed rate $V_{\text {st }}$ representing the minimum of the different limitation types is called: modified static look ahead.

$V_{\mathrm{st}}=\min \left(V_{\mathrm{fprog}}, V_{\mathrm{ftcy}}, V_{\mathrm{ft}}, V_{\mathrm{flsacc}}, V_{\mathrm{flstjerk}}, V_{\mathrm{jtcurv}}\right)$

The static look ahead well describes the machine dynamic behavior, but this feed rate calculation is done in costume time for the running block unaware of the following block (old machines). These problems are resolved by the integration of the dynamic look ahead (modern machines), in such a way that the NCU becomes able to anticipate the trajectory for the next 200 blocks. In fact, with the anticipation, we can avoid the overtaking caused by violent decelerations.

\subsubsection{Dynamic look ahead}

The term "dynamic" means that we need to calculate the speed profile with its two deceleration/acceleration phases on the running block. The dynamic look ahead permits then to anticipate in speed and in acceleration, thanks to the knowledge of the next blocks. In the case of any speed profile, the modeling is diagrammed in Fig. 6.

$>$ Calculation model of the acceleration and deceleration distance

The NC tool machine axis does not allow the same acceleration distance, according to the speed thresholds

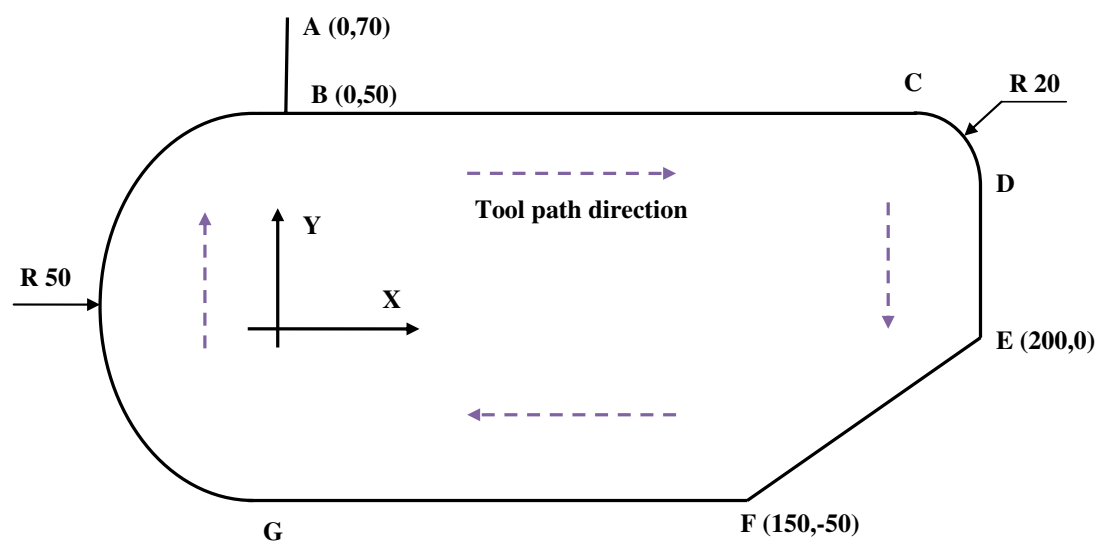


Fig. 9 Machining strategy in convergent parallel Spirals (Mastercam $\odot$ )

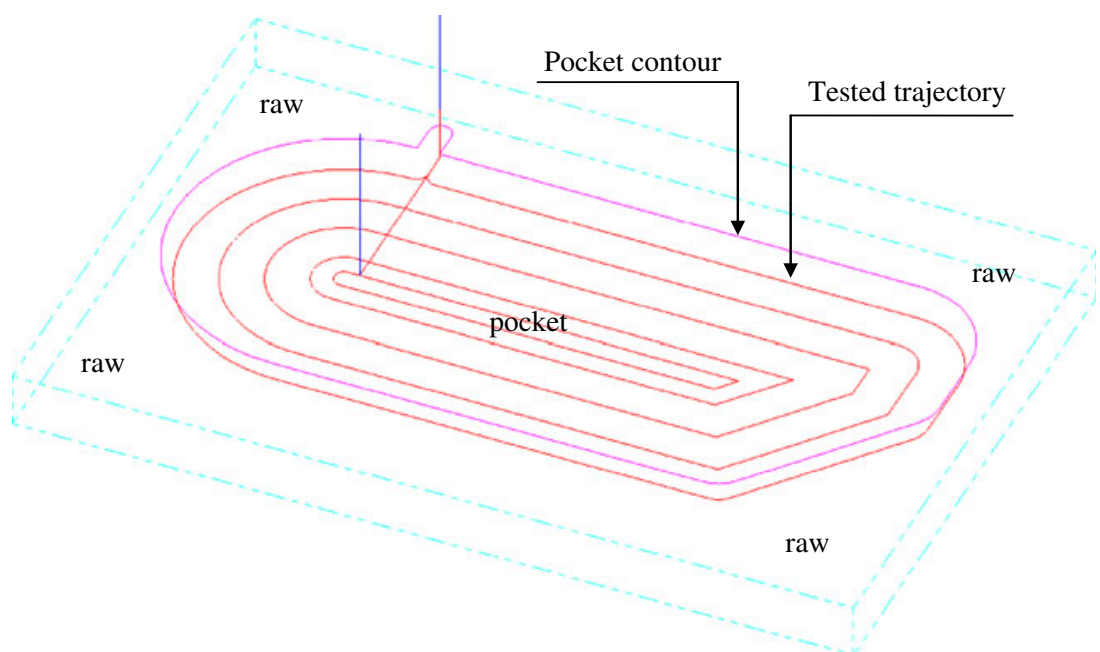

where it is located. For example, the distance covered by the machine is shorter if it accelerates from 13 to $15 \mathrm{~m} / \mathrm{min}$, comparatively to an acceleration of 11 to $13 \mathrm{~m} / \mathrm{min}$. To reach the programmed speed, the machine covers the acceleration distance $d_{\mathrm{acc}}$ during the time $t_{\mathrm{cy}}$. Likewise for the deceleration, in order to cancel the feed rate or to diminish it, the machine covers the deceleration distance $d_{\text {dec }}$ during the time $t_{\text {cy }}$ according to the following Eq. 20:

$V_{\mathrm{fprog}}=\frac{d}{t_{\mathrm{cy}}}$

Pateloup [13] shows that in HSM conditions, if the distance to cover between two successive information is inferior to $3 \mathrm{~mm}$, then the feed rate falls until $50 \%$. For block $i$, the model of the distances $d_{\mathrm{acc}}$ and $d_{\mathrm{dec}}$ is the following:

$d_{\mathrm{acc}}(i)=\left(V_{\mathrm{f}}(i)-V_{\mathrm{f}}(i-1)\right) \times t_{\mathrm{cy}}$

$d_{\mathrm{dec}}(i)=\left(V_{\mathrm{f}}(i)-V_{\mathrm{f}}(i+1)\right) \times t_{\mathrm{cy}}$

A HSM machine is made up of several components (axis, controller...) having their own limits and which interact between themselves. This makes the machine modeling dependent on several parameters.

The structured dynamic modeling will be programmed with Matlab $(C)$ in order to develop a feed rate simulator. This is the objective of the following part.

\section{HSM machining simulation}

In a first stage, we are determined to simulate the milling phenomena put at work. In a second period, tests on machines are realized so as to correlate the simulations with the reality.

In order to study the influence of the HSM machine dynamic behavior in milling, we start by developing the CAM model and the generation of a NC file. Then, we simulate the tool trajectories with Matlab $\odot$ by using the NC file already developed by the CAM model. Afterwards, we represent the corresponding feed rate profiles in static look ahead and in dynamic look ahead. Finally, we compare the simulated feed rate profiles with those of the experimental statements.

\subsection{Simulation step}

The step is represented in the following diagram (Fig. 7):

This step includes five main stages:

1. Conceive the part in CAD with SolidWorks $\odot$.

2. Develop the part CAM model with Mastercam(C).

3. Generate the NC file useful for the simulation tool.

4. Integrate the dynamic models:

4.1 Trajectory preparation by using the trajectory Interpolation Tolerance TIT.

4.2 Calculation of the static look ahead type on the trajectory (Ac/deceleration/jerk/feed rate. maxi. / axis + interpolation cycle time).

4.3 Calculation of the dynamic look ahead type on the trajectory (Ac/deceleration/jerk.. maxi. /axis + look ahead).

4.4 Storage of speed constraints for each block (static look ahead).

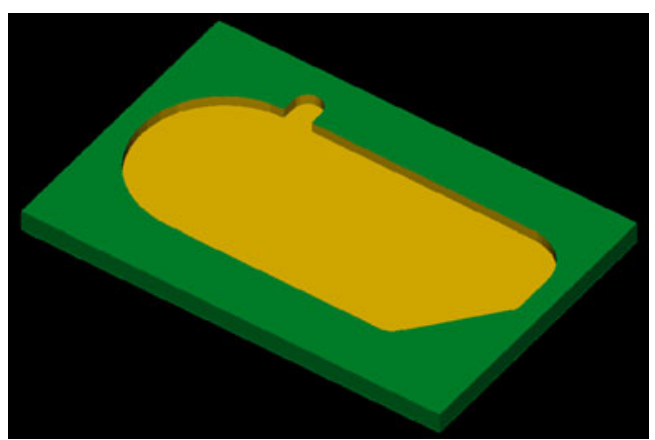

Fig. 10 Pocket obtained by Mastercam ( 
Fig. 11 Tool path simulated with Matlab(C)

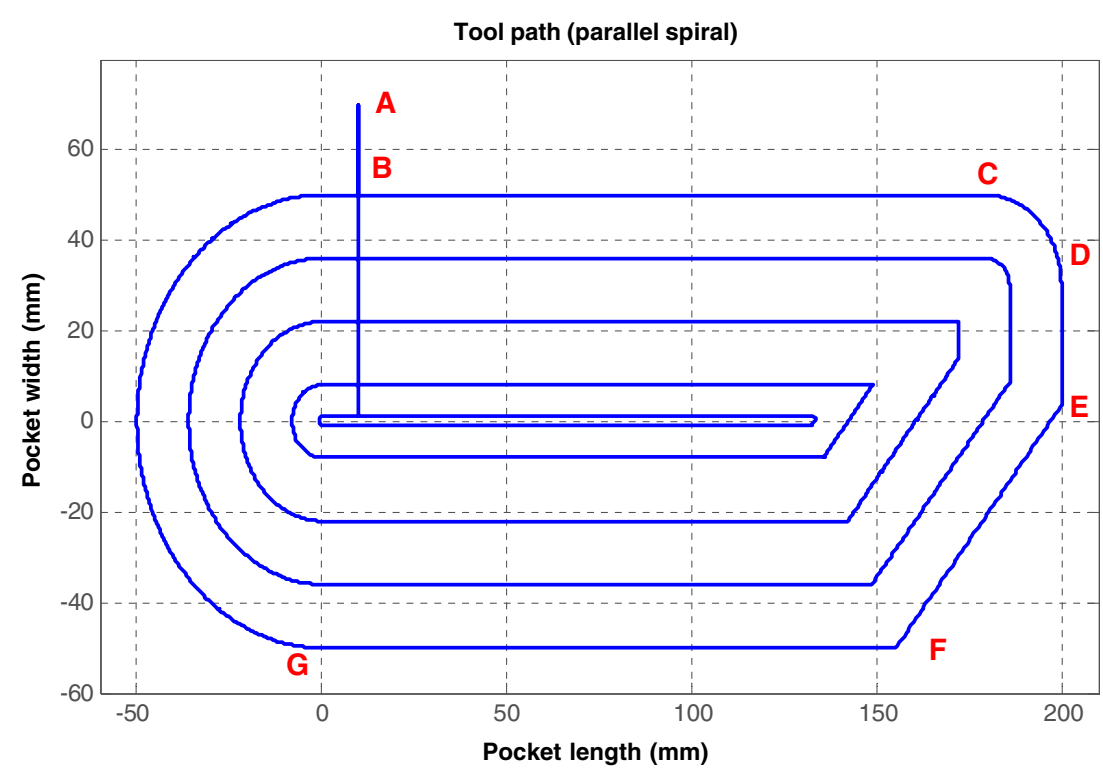

4.5 Storage of the instruction of the imposed anticipated speed at each axis according to the curvilinear abscissa (dynamic look ahead).

5. Make the speed (feed rate) profile with Matlab $\subset$ software.

3.2 Machining simulation of a complex shape test pocket

\subsubsection{Pocket definition}

A trajectory proposed by Cherif (Fig. 8) [15] has been tested on a HSM machining center (IUT of Nantes / HERMLE C800U 5 axis-HENDENHAIN TNC430-Acceleration $5 \mathrm{~m} / \mathrm{s}^{2}$ ), for two programmed feed rates from 15 to $35 \mathrm{~m} /$ min. The tests have permitted to record the real feed rate during the time. A confrontation between the simulation results and the experimental results will be realized in order to validate the developed simulation model.

\subsubsection{Hollowing out, strategy, and pocket characteristics}

\section{$>$ Hollowing out and strategy}

The rough piece is prismatic of dimensions $(300 \times 200 \times$ 20 ), the axial pass depth is of $10 \mathrm{~mm}$. The tool used is a double cut milling tool of a diameter $\varnothing 20$. The other parameters are taken by default.

Figure 9 presents the rough piece, the pocket, and the hollowing out strategy in convergent parallel spirals.

The final pocket obtained by Mastercam $\odot$ is presented in 3D in Fig. 10.

$$
>\text { Pocket characteristics }
$$

Fig. 12 Modified tool trajectory

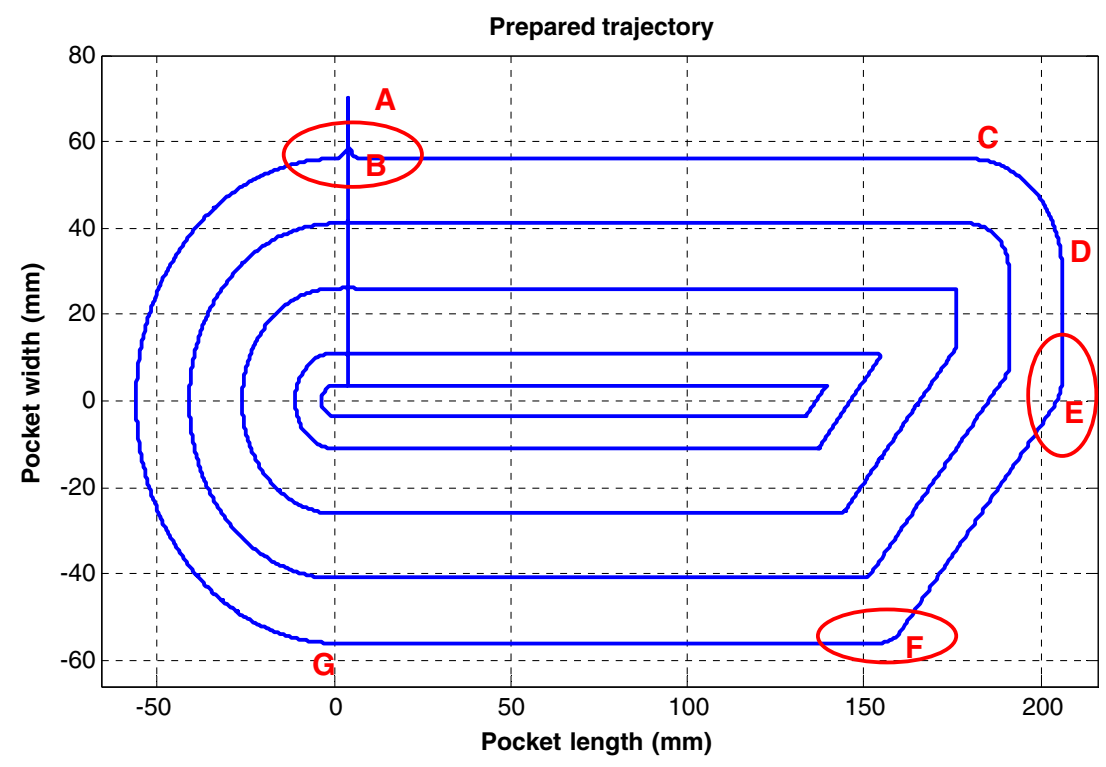



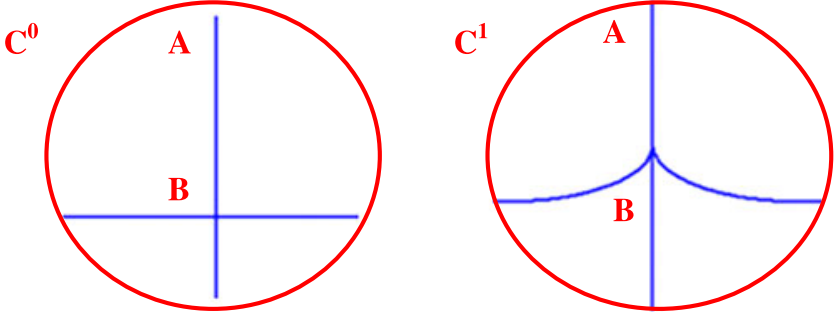

Fig. 13 Zoom of the passage from $C^{0}$ to $C^{1}$ on the segment $A B$

The geometry of this pocket allows us to test the interpolation G1, G2 and to solicit one or several axis simultaneously:

* Test the monoaxial linear interpolation G1 (portions $\mathrm{AB}, \mathrm{BC}, \mathrm{DE}$, and $\mathrm{FG}$ of the trajectory tested on the first machining pass (Fig. 9)).

* Test the bi-axial linear interpolation G1 (EF).

* Test the circular interpolation G2 (the long radius GB and the small radius $\mathrm{CD} \ldots$..)

* Test the tangencial discontinuities (of speed) of axis change (point B...).

* Test the curvature discontinuities (of acceleration in C, D and G points...)

\subsubsection{Real feed rate simulation}

$>$ Trajectory definition

We start with the tool path simulation representing the pocket hollowing out operation. It is realized with Matlab $(\mathcal{C}$ after the reading of the Mastercam $(\mathrm{C}$ NC file (Fig. 11).

The total length of the pocket machining trajectory $L_{\text {traj }}$ is calculated by the numerical simulator. $L_{\text {traj }}=2,300.7 \mathrm{~mm}$.
Then, we pass on to the definition of trajectory using the trajectory interpolation tolerance TIT $(4 \mu \mathrm{m})$. This step is done by the integration of an arc of circle at each tangential discontinuity.

The tool path, defined while sweeping the pocket, consists in integrating arcs of circles of small radius calculated following the Eq. 1 and which are between 1.9 and $11 \mu \mathrm{m}$. So as to make these arcs visible, they have been voluntary exaggerated on Fig. 12. Besides, the arcs added to the first pass are circled in red.

Figure 13 presents a zoom at the level of the segment $\mathrm{AB}$ before and after the integration of the arcs - the passage from the $\mathrm{C}^{0}$ discontinuity to the $\mathrm{C}^{1}$ discontinuity.

$>$ Calculation of the Static Look Ahead type on the trajectory

The integration of the dynamic behavior in the simulation is carried out by the calculation of the type static Look Ahead.

The tool feed rate profile influenced by the HSM Hermle machining center (capacity of axis, discontinuities...) is simulated under Matlab $\odot$. This simulation corresponds to the CAM pocket machining for the programmed speed $15 \mathrm{~m} / \mathrm{min}$ (Fig. 14).

The feed rate is detailed over four areas in the following figure:

(Figure 15)

The static look ahead describes the machine dynamic behavior. This is visible in the difference between the programmed and the real feed rate. This difference will be more obvious and will be examined in the feed rate profile generated with the dynamic look ahead.

$>$ Calculation of the Dynamic Look Ahead type on the trajectory
Fig. 14 Simulated feed rate profile (Static Look Ahead) on the whole pocket

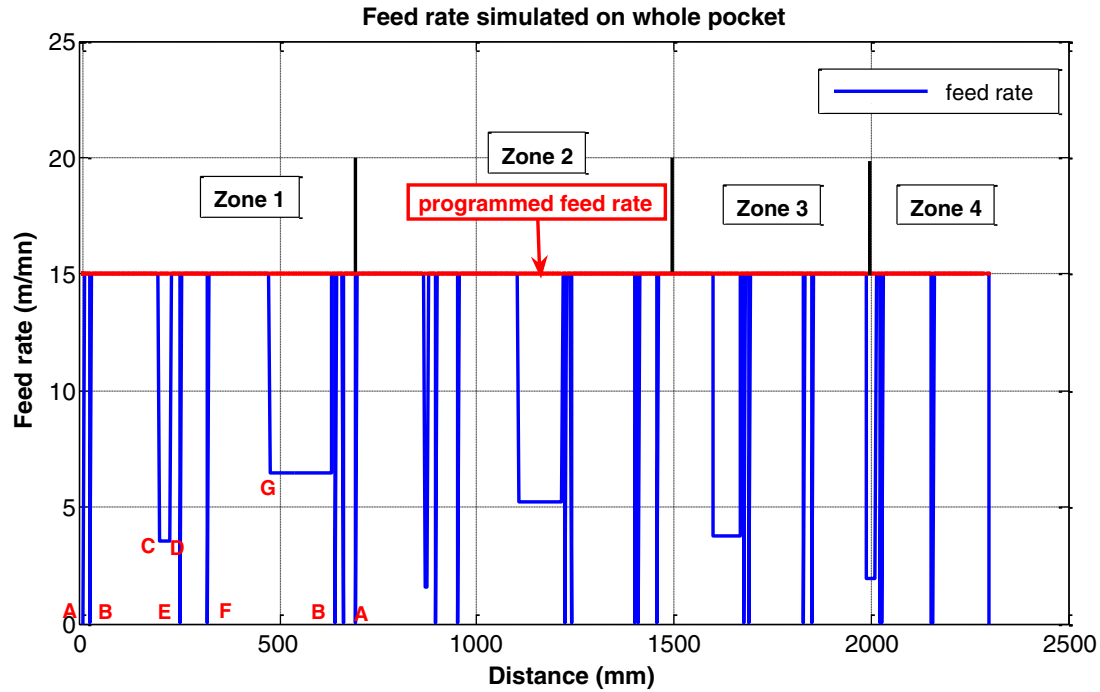



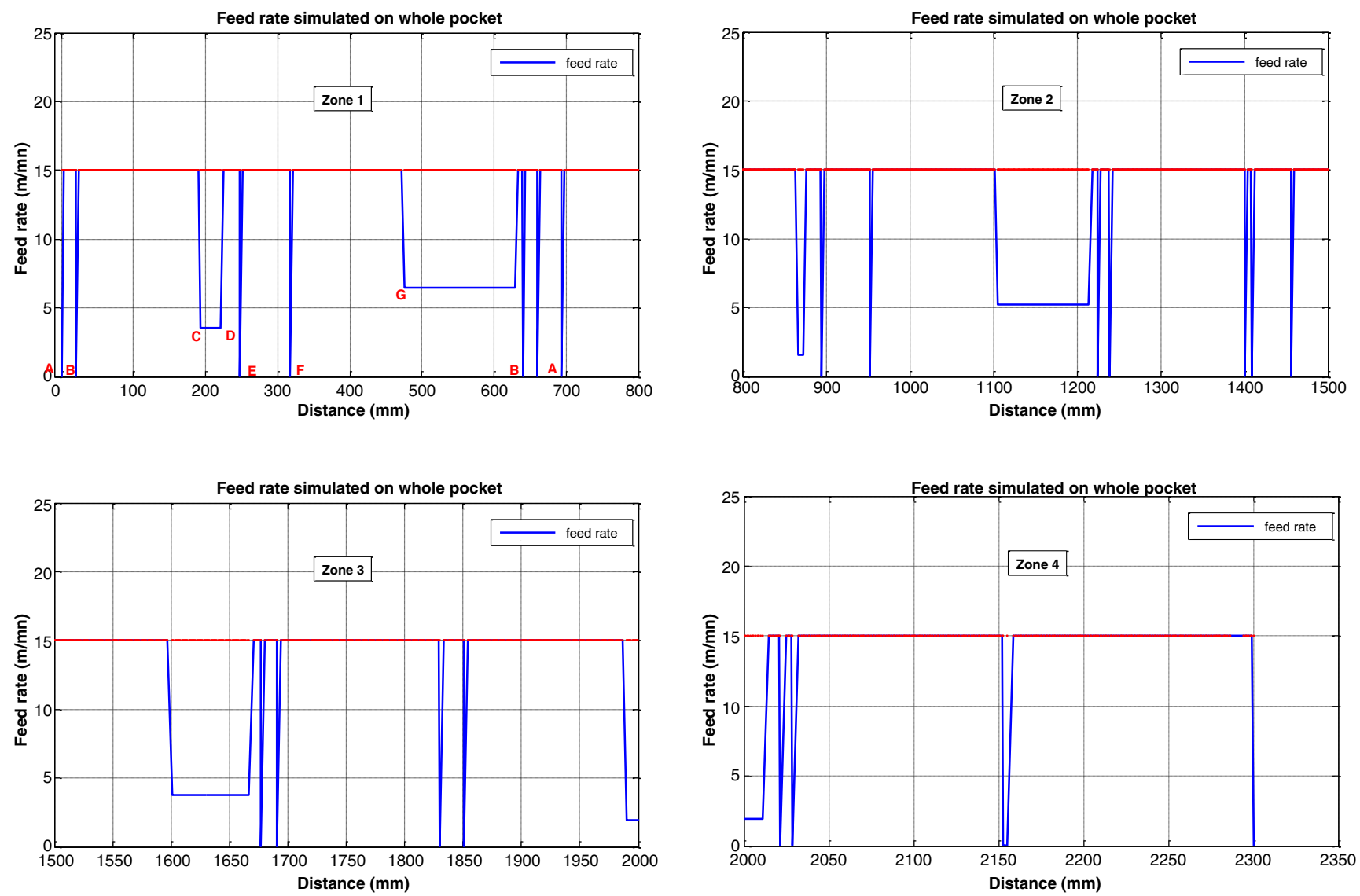

Fig. 15 Simulated feed rate profile (static look ahead) detailed over four areas

The dynamic look ahead allows to anticipate in speed and in acceleration. Thanks to the knowledge of the following blocks, the NCU calculates the speed profile with its acceleration and deceleration phases on each block, taking into account the speed profile in the next block. A calculation model of the acceleration and deceleration distance of each block, by taking into account the preceding and the following speeds, is integrated in our simulator.

In fact, with the anticipation, we can avoid the overtaking caused by the violent decelerations. The feed rate profile, simulated with the dynamic look ahead and the parameters of HSM machining center Hermle C800U 5 axis (jerk $0.5 \mathrm{~m} / \mathrm{s}^{3}$ ), on the trajectory ABCDEFG (Fig. 11), is shown in Fig. 16. Then a comparaison with the experimental statement (Fig. 17) for the same trajectory tested on the HSM MC Hermle, is realized with the same programmed feed rate $15 \mathrm{~m} / \mathrm{min}$.

We notice the influence of the anticipation on the speed profile in comparison to that of the static look ahead. The decelerations are known in advance and are carried out on the current blocks. That really describes the recent HSM dynamic machines behavior.
The speed profile simulated (Fig. 16) on the test trajectory (Fig. 8 ) is very near ( $2 \%$ of error) to the profile of the experimental statement (Fig. 17).

On the $\mathrm{C}^{0}$ discontinuities at the points $\mathrm{B}, \mathrm{E}$, and $\mathrm{F}$, the feed rate is very near to 0 . These speed limitations are due to the passage of the tool by the small connection radius. More precisely, the speed is limited by the machine

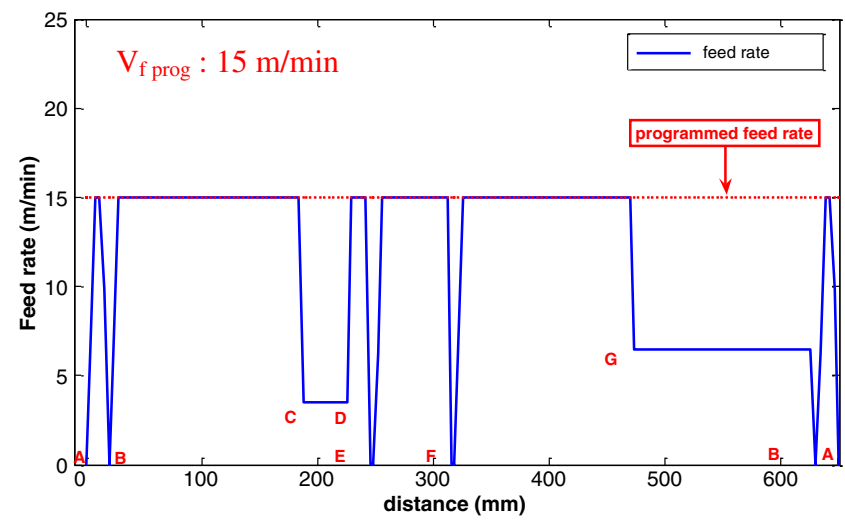

Fig. 16 Real feed rate profile simulated with the dynamic look ahead on the trajectory ABCDEFG 


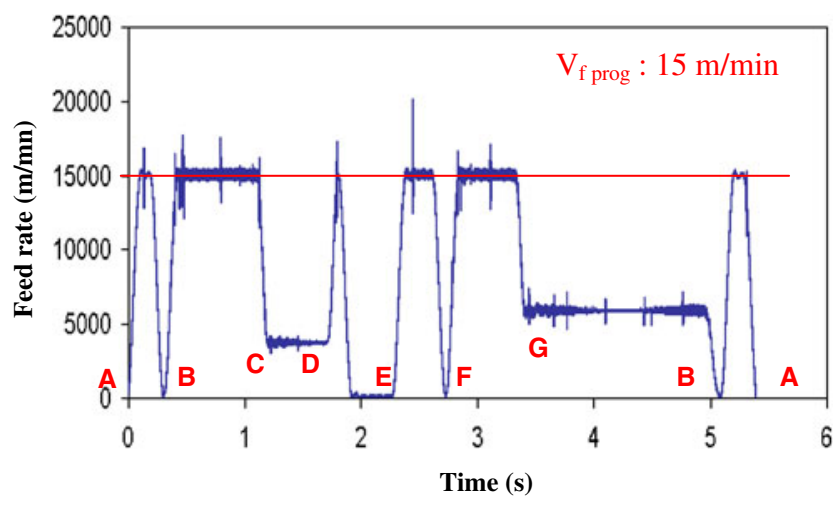

Fig. 17 Feed rate profile recorded experimentally [15]

maximal jerk. For the $\mathrm{C}^{1}$ discontinuities at the portions $\mathrm{CD}$ and $\mathrm{GB}$, the speed limitations on the circular portions are independent from the programmed speed. For the rest of the profile (Fig. 14), we have clearly seen that the speed continues to diminish for each pass at the portions $\mathrm{CD}$ and GB. That is due to the continuous arc diminutions. Therefore, the controller imposes these limitations according to the HSM machine axis characteristics and mainly the maximal jerk.

\subsection{Machining Simulation and experimental validation} of a complex part with connections

\subsubsection{Part definition}

We have developed the simulation of the tool trajectory and of the machining feed rate (in linear interpolation G1) of a piece having two curvatures, one convex, and one concave (Fig. 18). Thus, we have succeeded in confronting the experimental results recorded by the CMAO team in terms of speed profiles, following $\mathrm{X}$ and $\mathrm{Z}$, to validate our

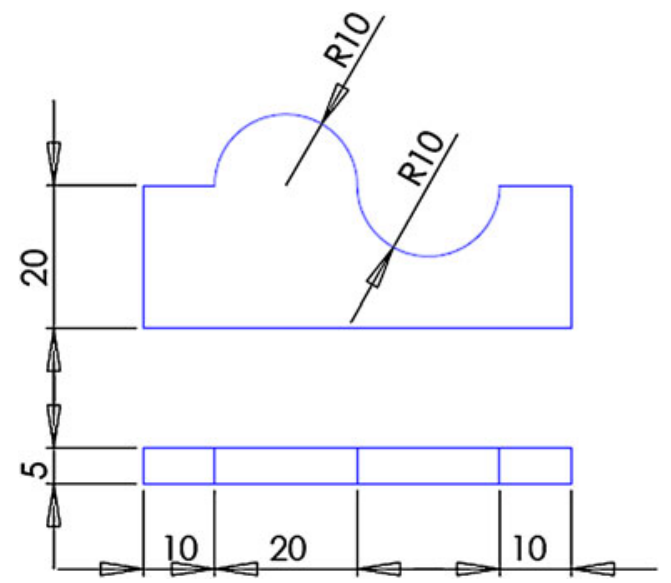

Fig. 18 The drawing of a part definition
Table 1 The three-axis Huron KX10 HSM machine characteristics

\begin{tabular}{|c|c|}
\hline Tool-Machine & Characteristics \\
\hline Work volume & $(1000) \times(700) \times(550) \mathrm{mm}$ \\
\hline Maximal feed rate & $\begin{array}{l}30 \mathrm{~m} / \mathrm{min}(X, Y) \text { and } 18 \mathrm{~m} / \mathrm{min}(Z) \text { in } \\
\text { rapid } 10 \mathrm{~m} / \mathrm{min}(X, Y, Z) \text { in } \\
\text { programmable work }\end{array}$ \\
\hline Maximal acceleration & $5 \mathrm{~m} / \mathrm{s}^{2}(X), 5 \mathrm{~m} / \mathrm{s}^{2}(Y), 3 \mathrm{~m} / \mathrm{s}^{2}(Z)$ \\
\hline Maxi. Jerk & $50 \mathrm{~m} / \mathrm{s}^{3}(X, Y, Z)$ \\
\hline $\begin{array}{l}\text { Interpolation cycle time } \\
\text { (tcy) }\end{array}$ & $2 \mathrm{~ms}$ \\
\hline $\begin{array}{l}\text { Trajectory Interpolation } \\
\text { Tolerance (TIT) }\end{array}$ & $7 \mu \mathrm{m}$ \\
\hline Look Ahead & 100 lines \\
\hline
\end{tabular}

simulation model. The machining is carried out on the three axis HSM Huron KX10 machine with NCU siemens 840D.

\subsubsection{Machine technical characteristics}

The machine technical characteristics (HSM Huron KX10 3 axis) are detailed in Table 1.

\subsubsection{The tool trajectory simulation}

By applying the diagram stages of Fig. 7, the part is conceived on the software CAD Solid Works $\mathbb{C}$, then exported as IGES file towards the software CAM Mastercam $($ ), where a machining simulation with a ball-end cutter of Ø20 in diameter is realized (Fig. 19).

Then, the NC file is sent to the simulation software Matlab $@$, where the tool trajectory is simulated (see the first pass of go and back trajectory simulated in the Fig. 20).

\subsubsection{Feed rate profile simulation and validation} with the experimental result

$\gg$ Integrated arcs of circle model with compaction $\left(\mathrm{C}^{1}\right.$ trajectory)

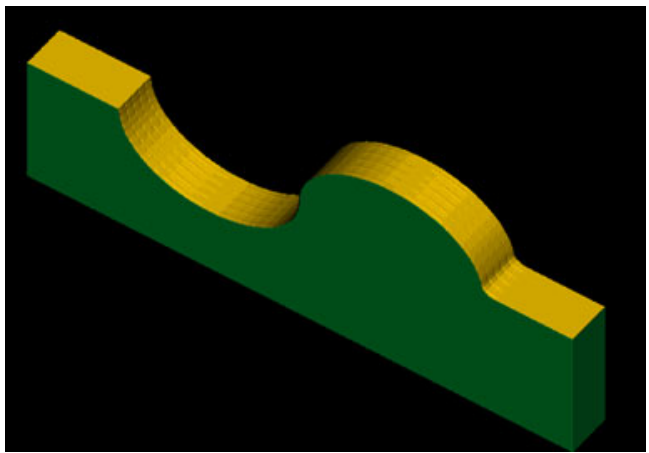

Fig. 19 Virtual piece obtained by Mastercam $\subset$ 


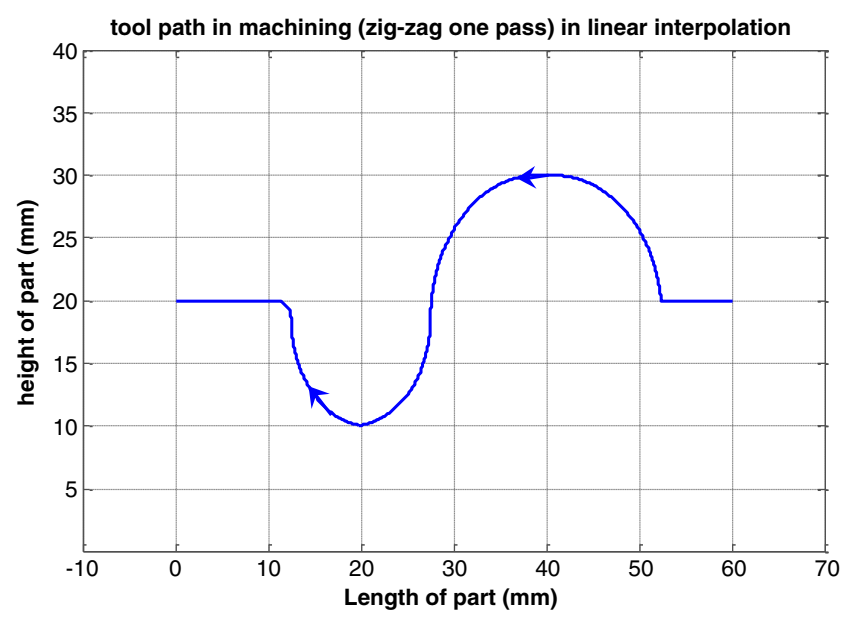

Fig. 20 A go-and-back simulated trajectory

The feed rate profile generation in linear interpolation on Matlab $($ passes then by the dynamic modeling of the NCU of the HSM machine. One of the crossing models of tangential discontinuities is the integration of arcs between two linear blocks. Since the whole part machining trajectory is in linear interpolation, we adopt this model to estimate the NCU behavior, badly mastered during the passage of the tangential discontinuities. Besides, the NCU achieves the blocks compaction of small blocks into $5 \mathrm{~mm}$ segments. Afterwards, we are going to simulate the feed rate profile and compare it with that of the experimental statement.

\section{$>$ Feed rate profile}

Figure 21 presents the simulated and the measured feed rate profiles. The experimental statement speed profile for the Huron KX10 is measured for a set speed of $9.6 \mathrm{~m} / \mathrm{min}$.

Comparison of the simulation result and the experimental statement
We have simulated a speed profile by the arcs of circle integration model, with compaction. This profile is very near $(5 \%$ of error) the experimental statement profile. But, there are some differences caused by a slight trajectory discretization. Hence, we notice that the compaction has conserved a more important value of the feed rate, for it consists in compacting the blocks of small segments which provoke the slowing down of the machine, imposed by the interpolation cycle time $t_{\mathrm{cy}}$. If we have less compaction, we will get more slowing down and consequently a greater machining time.

\section{Conclusion}

In this article, we have been interested in machining simulation of a pocket and of a complex profile. The objective is to introduce the dynamic modeling of the HSM machine in the simulation of a given trajectory.

In a first step, we have detailed the HSM machining center dynamic modeling. Then, we have explained the passage of the CAM model towards numerical simulation software, passing through the modeling. In a second step, we have simulated the real feed rate of a pocket hollowing out.

In the second part, we have been interested in the feed rate simulation of a complex part machining, in order to show the anticipation influence with compaction. We have discovered that the speed is variable according to the shape to be machined and the anticipation keeps a more important speed evolution.

Afterwards, we come to the conclusion that the simulation tool which permits to give values very near the reality ( $\leq 5 \%$ of errors) for the pocket hollowing out and for the complex part machining.
Fig. 21 Feed rate profile simulated and measured on machine

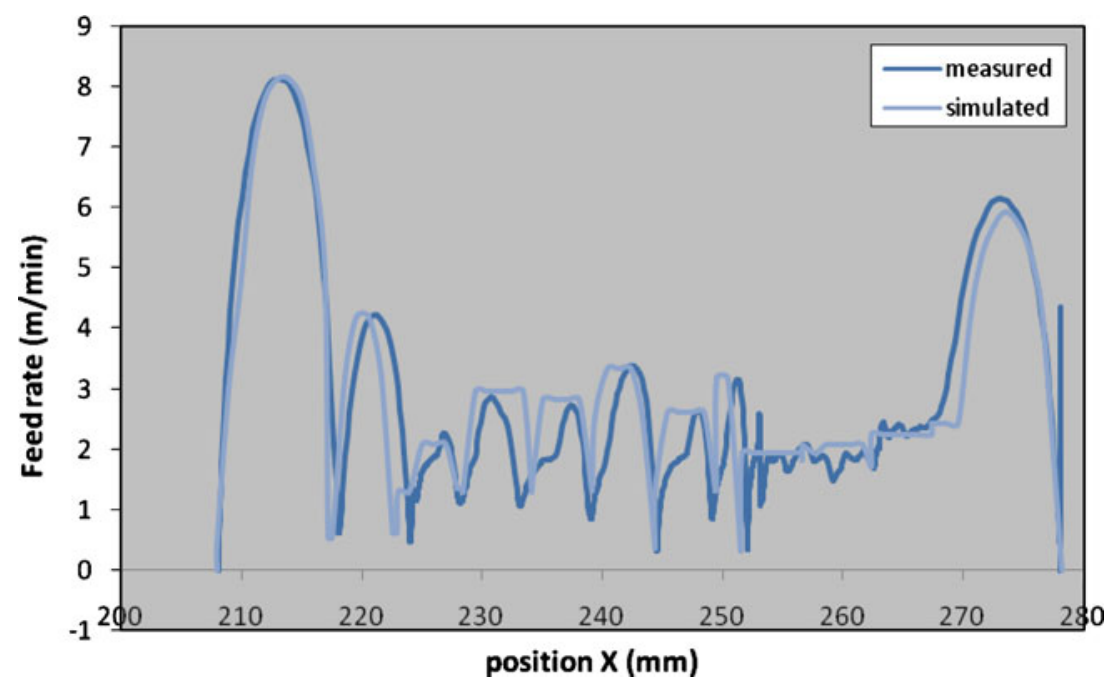


The real feed rate profile indicates that several parameters must be put into evidence to optimize the complex shapes machining. Hence, in order to make an optimal choice of a machining strategy, all we have to do is to analyze the different critical criteria.

Open Access This article is distributed under the terms of the Creative Commons Attribution Noncommercial License which permits any noncommercial use, distribution, and reproduction in any medium, provided the original author(s) and source are credited.

\section{References}

1. Tournier C, Lavernhe S, Lartigue C (2005) Optimisation en fraisage 5 axes grande vitesse, CPI'2005, CDRom paper. Casablanca, Morocco, pp 1-11

2. Blanchard N, Rabany T, Duc E (2005) Lobes de stabilité en UGV approche expérimentale en usinage de poches. J Mécanique Ind 6:411-415, France

3. Bouaziz Z, Ben Younes J, Zghal A (2004) Methodology of machining costs evaluation for die and mould manufacturing. $J$ Mater Process Technol 152:237-245

4. Monreal M, Rodriguez CA (2001) Influence of tool path strategy on the cycle time of high speed milling. J Comput-Aided Des 35:395-401

5. Terrier M, Dugas A, Hascoet J-Y (2004) Qualification of parallel kinematics machines in high-speed milling on free form surfaces. Int J Mach Tools Manuf 44:865-877

6. Mawussi K, Lavernhe S, Lartigue C (2003) Usinage de poches en UGV. Aide au choix de stratégies. Int Rev Cfao Graphic Inf Technol 18(3):337-349
7. Tapie L, Mawussi KB, Anselmetti B (2006) Machining strategy choice: performance viewer. IDMME 2006, CDROM paper, Grenoble France, May 17-19

8. Guardiola A, Rodriguez CA, Souza AF, Dos Santos MT (2007) Influence of tool path interpolation on cycle time and following error during hight-speed milling of die and mold surfaces. Sixth International Conference on HIGH SPEED MACHINING

9. Souza AF, Coelho RT (2007) Investigation of tolerances required for NC program's generation using spline polynomial and linear interpolation to describe a free form tool path for hight speed milling. Sixth International Conference on HIGH SPEED MACHINING.

10. Dugas A (2002) CFAO et UGV, Simulation d'usinage de formes complexes, Thesis of doctorate of central school of Nantes (France), 13 December

11. Pateloup V (2005) Amélioration du comportement cinématique des machines outils UGV. Application au calcul de trajets d'évidement de poches, Thesis of doctorate of doctoral school of Blaise Pascal-Clermont II university, Blaise Pascal (France), May

12. Pateloup V, Duc E, Pascal R (2004) Corner optimization for pocket machining. Int J Mach Tools Manuf 44:1343-1353

13. Pateloup V (2002) Usinage à grande vitesse des poches; prise en compte du comportement dynamique de la machine outil dans le calcul de la trajectoire, DEA Memory of Automated Production, Superior Normal School of Cachan (France), September

14. Tapie L, Mawussi KB, Anselmetti B (2006) Circular tests for HSM machine tools: bore machining application. Int J Mach Tools Manuf 47:805-819

15. Mehdi chérif (2000) Reconstruction d'un modèle CAO à partir des mouvements réels d'une machine MOCN, master Memory, IRCCyN, central school of Nantes (France), 29 september 